\title{
Canavanine Resistance and the Mechanism of Arginine Uptake in the Fission Yeast Schizosaccharomyces pombe
}

\author{
By PETER A. FANTES* AND JAMES CREANOR \\ Department of Zoology, University of Edinburgh, Edinburgh EH9 3JT, UK
}

(Received 26 April 1984; revised 24 July 1984)

\begin{abstract}
The mechanism of resistance to the arginine analogue L-canavanine, and of arginine uptake, were examined in the fission yeast Schizosaccharomyces pombe. Two mutants with increased resistance to canavanine were analysed genetically: both were double mutants, and in each case one mutation conferred resistance to canavanine, while the other enhanced this resistance. Evidence is presented that can 1.1 strains are defective in one system for arginine uptake, which presumably prevents entry of canavanine into the cell. This system operates in the wild-type whether the nitrogen source supplied is ammonium or glutamate. Double mutants carrying canl.l and an arginine requirement are unable to grow on ammonium medium even when supplied with exogenous argine, while growth can occur on glutamate plus arginine. This suggested the existence of a second uptake system for arginine which is absent during growth on ammonium, and direct measurements of the rates of arginine uptake under various conditions confirmed this. Our observations closely parallel those made on the budding yeast Saccharomyces cerevisiae. The ability to select for or against function of the canl gene should facilitate certain types of genetical analysis in $S$. pombe.
\end{abstract}

\section{INTRODUCTION}

The fission yeast Schizosaccharomyces pombe is one of the best-characterized eukaryotic micro-organisms for genetic studies. In addition to the methods of classical genetics, recent advances (Beach \& Nurse, 1981) have stimulated interest in the use of recombinant DNA procedures combined with transformation of $S$. pombe. In contrast, the biochemistry and physiology of $S$. pombe lags behind that of the budding yeast Saccharomyces cerevisiae. In particular, no mutants have been reported in which a specific uptake system is genetically defective, though more general uptake defects have been described (Johnston \& Coddington, 1983). We have initiated an investigation into the genetic and physiological basis of resistance to the arginine analogue L-canavanine, for three main reasons. First, the genetics of cavavanine resistance have been investigated (Kohli et al., 1977) and resistant mutants are available. Second, a substantial body of information exists about canavanine resistance in Sacch.cerevisiae (for a review, see Cooper, 1982), and it is of interest to find whether the two distantly related yeasts are similar in this respect. Third, canavanine resistance may prove to be a useful genetic marker in S. pombe, since it may be selected or counter-selected, as in Sacch. cerevisiae (Whelan et al., 1979).

\section{METHODS}

Strains and genetical procedures. All strains used were derived from the wild-types $972 h^{-}$and $975 h^{+}$(Gutzet al., 1974). All the auxotrophs, and the canavanine resistant strains, were kindly provided by the Microbiology Institute of the University of Bern, Switzerland. Strain ED 428 was sent to us as 'canl.1 $h^{-}$, and strain ED 429 as 'can $2.1 \mathrm{~h}^{-}$. Crosses were carried out according to Gutz et al. (1974); stable diploid strains were constructed using the mat 2.102 mating type mutation as described by Kohli et al. (1977).

Media and culture conditions. EMM-A was essentially EMM3 (Creanor \& Mitchison, 1982), but with $2 \%(\mathrm{w} / \mathrm{v})$ glucose, and supplemented with $\mathrm{KCl}\left(1 \mathrm{~g} \mathrm{l}^{-1}\right)$. EMM-A contained ammonium chloride $\left(5 \mathrm{~g} \mathrm{l}^{-1}\right)$ as nitrogen source. 
EMM-G was identical to EMM-A but the nitrogen source was monosodium glutamate $\left(3.74 \mathrm{~g}^{-1}\right)$ in place of ammonium chloride. Solid media contained $2 \%(\mathrm{w} / \mathrm{v})$ Bacto-agar (Difco). Complex medium was YEA [yeast extract $0.5 \%(\mathrm{w} / \mathrm{v})$, glucose $3 \%(\mathrm{w} / \mathrm{v})$, Bacto-agar $2 \%(\mathrm{w} / \mathrm{v})]$. Growth supplements were included at $75 \mathrm{mg}^{-1}$ for the growth of auxotrophic strains. L-Arginine and L-canavanine were added as hydrochlorides: canavanine hydrochloride was added from a filter-sterilized stock to autoclaved medium. The growth and sampling of liquid cultures, and cell number estimation, were carried out according to Mitchison (1970). Strains were grown at $30^{\circ} \mathrm{C}$.

Arginine uptake. This was determined by adding to a sample (0.5-2 ml) of growing cells the desired amount of L[5(n)- ${ }^{3} \mathrm{H}$ ]arginine monohydrochloride (Amersham; $703 \mathrm{GBq} \mathrm{mmol}^{-1} ; 37 \mathrm{MBq} \mathrm{ml}^{-1}$ ) plus unlabelled L-arginine monohydrochloride and/or other amino acids as required. Samples were removed, filtered onto $2 \cdot 1 \mathrm{~cm} \mathrm{GF/C}$ filters (Whatman), and washed four times with unlabelled arginine hydrochloride solution (1 mg ml-1) and four times with water. The filters were dried and counted in $0.5 \%(\mathrm{w} / \mathrm{v})$ butyl PBD in toluene, on a Packard model 2407 liquid scintillation spectrometer. Counting efficiency was $35 \%$.

Chemicals. These were all of analytical grade.

\section{RESULTS}

The effect of various concentrations of canavanine on the growth of wild-type and canavanine resistant strains was investigated by measuring the diameter of colonies derived from single cells after $3 \mathrm{~d}$ growth. Two media were used, EMM-A containing ammonium chloride as nitrogen source and EMM-G containing monosodium glutamate. Table 1 shows the effect of different concentrations of canavanine on the wild-type 972, and the two canavanine resistant strains ED 428 (carrying can1.1) and ED 429 (carrying can2.1). Strain ED 460 is a derivative of ED 428 described below.

At all canavanine concentrations, the strains were more sensitive when grown with glutamate as nitrogen source than with ammonium. The use of glutamate has been reported (Strauss, 1979) to allow a higher rate of uptake of certain substrates, and this was presumably responsible for the increased sensitivity to canavanine. The wild-type 972 was completely inhibited at the lowest canavanine concentration tested during growth on glutamate. Under the same conditions both ED 428 and ED 429 showed substantial growth, though neither strain grew as well in the presence of canavanine at any concentration as in its absence (Table 1). During growth on ammonium medium, the wild-type and the mutants showed greater resistance to canavanine than during growth on glutamate. However the wild-type was substantially more sensitive than either ED 428 and ED 429 (Table 1). Despite the different levels of resistance shown by the three strains, the extent of inhibition was consistently greatest for the wild-type and least for ED 428 under both sets of conditions (Table 1).

The tests just described were based on the ability of single cells to proliferate and form colonies under the various conditions. For genetical analysis, a purely qualitative test was required so that strains could be easily and unambiguously classified as sensitive or resistant. For this, patches of cells growing actively under non-selective conditions were replicated onto agar medium containing glutamate as nitrogen source and canavanine at $10 \mu \mathrm{g} \mathrm{ml}^{-1}$. After 3-5 d incubation strains could be classified according to the degree of resistance.

The genetic basis of canavanine resistance in strains ED 428 and ED 429 was investigated by crossing each strain to the wild-type $975 \mathrm{~h}^{+}$and analysing the meiotic progeny by tetrad dissection. In both crosses the segregation of canavanine resistance was irregular, in contrast to the regular $2: 2$ segregation of the other marker in the cross, mating type. In any single tetrad, either 0,1 or 2 progeny were as resistant as the resistant parent. The remainder were either sensitive or showed some growth in the presence of canavanine after prolonged incubation. Varying the concentration of canavanine did not help to resolve this complex situation. The patterns of segregation led us to suspect that more than one gene with an effect on canavanine resistance was segregating, and prompted more detailed genetical analyses.

\section{Genetic analysis of ED 428}

Two progeny of the cross between ED 428 and wild-type were picked for further crosses. One of these, ED 428-1c, showed strong resistance similar to ED 428, while the other, ED 428-4d, showed weak resistance. 


\section{Table 1. Sensitivity of $S$. pombe strains to L-canavanine}

Strains were streaked onto the surface of agar medium (EMM-A has ammonium as nitrogen source, and EMM-G has glutamate), and incubated at $30^{\circ} \mathrm{C}$. After $3 \mathrm{~d}$ incubation the mean diameter of isolated colonies was estimated.

\begin{tabular}{|c|c|c|c|c|c|c|c|c|}
\hline \multirow[b]{3}{*}{ Strain } & \multicolumn{4}{|c|}{ Diameter $(\mu \mathrm{m})$ on EMM-A } & \multicolumn{4}{|c|}{ Diameter $(\mu \mathrm{m})$ on EMM-G } \\
\hline & \multicolumn{4}{|c|}{ Canavanine $\left(\mu \mathrm{g} \mathrm{ml}^{-1}\right)$} & \multicolumn{4}{|c|}{ Canavanine $\left(\mu \mathrm{g} \mathrm{ml}^{-1}\right)$ : } \\
\hline & 0 & 3 & 10 & 30 & 0 & 3 & 10 & 30 \\
\hline $972 h^{-}$ & 1000 & 150 & 100 & 60 & 1000 & s.c. & s.c. & s.c. \\
\hline ED 428 & 1000 & 700 & 700 & 700 & 1000 & 350 & 200 & 175 \\
\hline ED 429 & 700 & 400 & 350 & 250 & 700 & $150-300^{*}$ & $100-250^{*}$ & $50-125^{*}$ \\
\hline ED 460 & 1000 & 900 & 700 & 600 & 1000 & 200 & 75 & 50 \\
\hline
\end{tabular}

Table 2. Progeny types in crosses between canl.l and wild-type strains

Strains ED 428-Ic and ED 428-4d are progeny of the cross between ED 428 and $975 h^{+}$(see text). ED 428-Ic shows strong canavanine resistance; ED 428-4d shows weak resistance.

\begin{tabular}{|c|c|c|c|c|}
\hline \multicolumn{3}{|c|}{ Tetrad types*: } & \multicolumn{2}{|c|}{ No. of tetrads } \\
\hline $\mathbf{R}$ & $\begin{array}{c}\text { (R) } \\
\text { (R) typ }\end{array}$ & 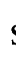 & ED 428-1c $\times 975 h^{+}$ & ED $428-4 d \times 972 h^{-}$ \\
\hline 2 & 0 & & 3 & 0 \\
\hline 1 & 1 & & 3 & 0 \\
\hline 0 & 2 & & 0 & 8 \\
\hline
\end{tabular}

The cross between ED 428-4d and $972 h^{-}$yielded only sensitive and weakly resistant progeny, showing a $2: 2$ segregation in each of eight tetrads analysed (Table 2). Microscopic examination of the cells showed that sensitive strains consisted of swollen and dead cells, while the weakly resistant progeny showed nearly normal cellular morphology after growth on canavanine.

In contrast, the cross between ED 428-1c and $975 h^{+}$gave an irregular segregation similar to that of the cross between ED 428 and $975 h^{+}$, with sensitive, weakly resistant and fully resistant progeny. The segregation pattern (Table 2) was consistent with the segregation of two genes controlling resistance to canavanine. One of these (canl.1) was presumably required for canavanine resistance, while the other (canx) interacted with the mutant can 1.1 allele to enhance the degree of resistance. On this basis ED 428-1c and the original resistant strain ED 428 were genotypically canI.I canx, since they both generated three resistance classes when crossed to a wild-type. In contrast ED 428-4d was canl. I can $x^{+}$since only sensitive and weakly resistant progeny resulted from a cross to wild-type. An alternative explanation, that the wild-type $975 h^{+}$ carried canx as a cryptic mutation, can be ruled out since a cross between an $h^{-}$, weakly canavanine resistant strain derived from the cross between ED 428-4d and $972 h^{-}$gave only sensitive and weakly resistant progeny when crossed to $975 h^{+}$. A twice-backcrossed derivative of ED 428 of genotype canl.1 canx ${ }^{+} h^{-}$was designated ED 460 (see Table 1) and was used as our standard can1.1 strain in further genetical and physiological experiments.

Canavanine is an arginine analogue, and one mechanism of resistance to it might be a defect in the uptake of the analogue and of arginine itself, by analogy with the better-defined situation in Sacch. cerevisiae (Grenson et al., 1966). To test this genetically, crosses were carried out between ED 460 and two arginine auxotrophs, $\arg 1.230$ and $\operatorname{arg6.238.~The~former~strain~is~unable~}$ to use ornithine in place of arginine, while the latter is able to do so ( $P$. Munz, personal communication). Two different auxotrophs were used to rule out any possible gene-specific interactions with canl.1.

The two sets of crosses gave essentially similar results (Table 3 ). The arginine requirement segregated $2: 2$ in each tetrad. Of the $\mathrm{Arg}^{+}$progeny, about half (type B) were resistant to 
Table 3. Progeny of crosses between ED 460 and arg $^{-}$strains

Crosses were (a) ED $460\left(\right.$ can $\left.1.1 h^{-}\right) \times \operatorname{argl} 1.230 h^{+} ;(b)$ ED $460 \times \arg 6.238 h^{+}$. Eight tetrads were dissected for each cross, and pooled data are shown.

$\begin{array}{cccccccccc}\begin{array}{c}\text { Progeny } \\ \text { type }\end{array} & \overbrace{\begin{array}{c}\text { Cross } \\ (a)\end{array}}^{\text {No. of progeny }} & \begin{array}{c}\text { Cross } \\ (b)\end{array} & & \text { EMM-G } & \begin{array}{c}\text { EMM-G } \\ + \text { arg }\end{array} & \text { EMM-A } & \begin{array}{c}\text { EMM-A } \\ + \text { arg }\end{array} & \begin{array}{c}\text { EMM-G } \\ + \text { can }\end{array} & \overbrace{\text { canl.I }}^{\text {Erowth* on: }} \\ \text { A } & 10 & 9 & + & + & + & + & - & + & + \\ \text { B } & 6 & 7 & + & + & + & + & + & - & + \\ \text { C } & 6 & 7 & - & + & - & + & \text { ND } & + & - \\ \text { D } & 10 & 9 & - & (+) & - & - & \text { ND } & - & -\end{array}$

* Scoring: - , no growth: (+), poor growth; + , strong growth; ND, not determined.

canavanine and about half (type A) sensitive (Table 3). The $\mathrm{Arg}^{-}$progeny could be tested for canavanine resistance, since inclusion of arginine in the medium to permit growth abolished the inhibitory effect of canavanine. However, two types of $\mathrm{Arg}^{-}$progeny could be distinguished. One type (type $\mathrm{C}$ in Table 3) grew on arginine supplemented medium as well as $\mathrm{Arg}^{+}$strains, irrespective of the nitrogen source. The other type (D in Table 3 ) grew poorly on EMM-G supplemented with arginine, and not at all on EMM-A supplemented with arginine. In addition, type D strains grew poorly on complex medium. It is very likely that the type D strains are arg can 1.1, since their phenotype resembles neither of the parents and is not wild-type. Detailed inspection of the segregation pattern within each tetrad, and backcrosses of putative double mutants (not shown) showed this supposition to be correct. Thus the double mutant strains (arg can 1.1) demonstrate an interaction between the two mutations at the phenotypic level. This is strongly reminiscent of the situation in Sacch. cerevisiae where canl arg double mutants were unable to grow even when provided with arginine under conditions of nitrogen repression (Whelan et al., 1979; Grenson \& Hou, 1972). The gene CAN1 in Sacch. cerevisiae is known to code for a specific permease concerned with the uptake of arginine and other basic amino acids (Grenson et al., 1966), and this prompted us to propose and test directly a model for arginine uptake and canavanine resistance in $S$. pombe. The model proposes the existence of two systems which are able to mediate the uptake of arginine. One of these (system I) shows some specificity for arginine, and is the major entry route for canavanine. The other system (system II) also allows the uptake of arginine. System II is repressed or inhibited by the presence of ammonium in the medium. This model explains all the observations described so far if the can $^{+}$gene codes for a component of system I. In can1.l strains canavanine is unable to be taken up and the cells are resistant to its inhibitory effects. Double mutants ( $\mathrm{canl} .1 \mathrm{arg})$ need to assimilate arginine from the medium in order to grow. This is possible when the nitrogen source is glutamate, when arginine enters by system II, but impossible during growth on ammonium since system II is ammonium inhibited. Direct tests of this model are presented below.

\section{Chromosome mapping of can1}

During the construction of canl strains also bearing a leul.32 mutation, linkage between can 1 and $l e u 1$ was fortuitously discovered. Of 20 tetrads analysed, 10 were parental ditype and 10 tetratype, suggesting a map distance of about 25 map units. This was confirmed by the analysis of 386 random spores, which gave a map distance of 25.4 map units. Three-point crosses including canl, leul and either tps 13.24 or $c d c 10.129$ demonstrated that canl was linked to tps 13 but not to $c d c 10$. The distance between canl and tps13, a centromere-linked marker (Gygax \& Thuriaux, 1984) was 34 map units, based on 192 progeny. The canl gene may be a useful genetic marker since this region of chromosome II is otherwise rather sparsely covered.

\section{Analysis of ED 429}

Aberrant segregations for canavanine resistance were observed in the cross ED $429 \times 975 h^{+}$. Both $2: 2$ and $3: 1$ segregations for sensitive : resistant progeny were observed initially. Closer 


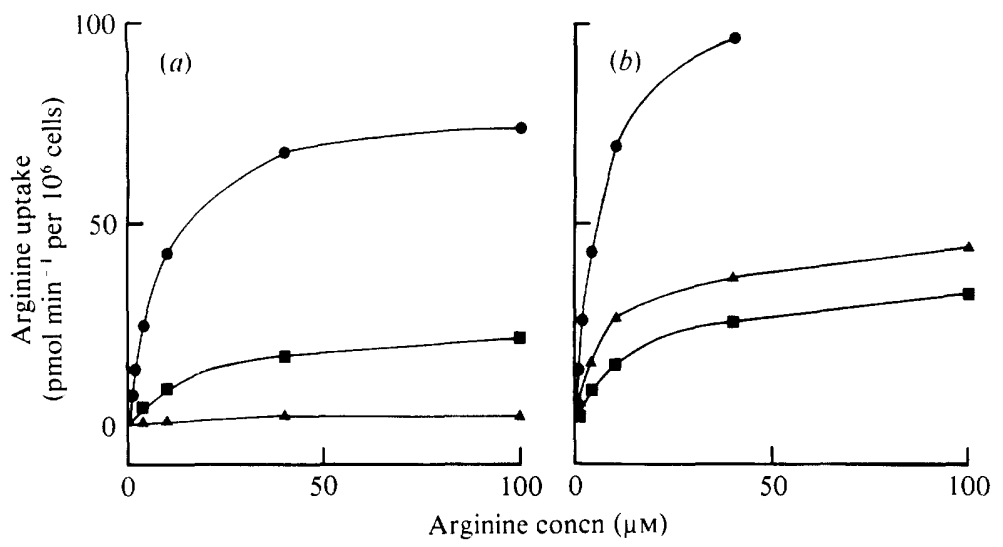

Fig. 1. Effect of arginine concentration on arginine upiake rates in $S$. pombe strains. Samples $(500 \mu l)$ of the exponential cultures indicated were added to $1 \mu \mathrm{Ci}\left[{ }^{3} \mathrm{H}\right]$ arginine and amounts of unlabelled arginine to bring the final concentration to those shown. Samples were incubated at $30^{\circ} \mathrm{C}$ for $8 \mathrm{~min}$, filtered, washed and counted as described in Methods. The counts were converted into pmol arginine taken up per min per $10^{6}$ cells, taking the counting efficiency as $35 \%$. (a) Cultures growing in EMM-A : $\mathbf{O}$, $972 h^{-} ; \mathbf{\Delta}$, ED 428; $\mathbf{\square}$, ED 429. (b) Cultures growing in EMM-G: O, $972 h^{-} ; \mathbf{\Delta}$, ED 428;, ED 429. Lineweaver-Burk plots of the uptake rates give the following values for $K_{\mathrm{m}}$ of the uptake system(s). For ammoniun-grown cells the $K_{\mathrm{m}}$ values are $10.5 \mu \mathrm{M}, 14.3 \mu \mathrm{M}$ and $20.0 \mu \mathrm{M}$ for 972, ED 428 and ED 429 respectively. For glutamate-grown cells the corresponding values are $7.7 \mu \mathrm{M}, 7.8 \mu \mathrm{M}$ and $13.8 \mu \mathrm{M}$.

scrutiny showed that of the three progeny initially classified as sensitive in each tetrad of the latter type, one showed a low level of canavanine resistance. This situation is analogous to that shown by ED 428, that is, it suggests the presence of an enhancer mutation in ED 429 . In crosses between enhancer-free can 2.1 and $\arg$ strains, no interaction was observed in double mutants, which grew well when supplemented with arginine, irrespective of nitrogen source. This suggested that can 2.1 was not intimately involved in arginine or canavanine uptake, although experiments described below point to some kind of uptake defect in can2.1.

\section{Arginine uptake by can strains}

Arginine uptake was determined for cells of 972, ED 428 and ED 429 during growth in EMMA (ammonium) or EMM-G (glutamate) media. Fig. 1 shows the rates of arginine uptake at various arginine concentrations. (Other experiments showed that the uptake was linear with time over the period used.) In all cases uptake approached saturation at $100 \mu \mathrm{M}$-arginine ; calculations based on Lineweaver-Burk plots (not shown) give $K_{\mathrm{m}}$ values between 7 and $20 \mu \mathrm{M}$ (see Fig. 1). The most striking observation was that strain ED 428 showed a much reduced uptake rate compared with the wild-type during growth in EMM-A. During growth in glutamate medium ED 428 showed a reduced uptake rate compared with 972 , though the relative reduction was only about $50 \%$ in this case. However, the wild-type rate in glutamate medium was higher than that in ammonium medium; in fact, the absolute difference in rates between ammonium and glutamate medium was nearly the same for both ED 428 and 972 . Expressed another way, the total rate of arginine uptake by 972 growing in EMM-G was additively composed of the rate of uptake in ammonium medium and an extra component. In ED 428 the uptake rate during growth in ammonium medium was very low, yet the 'extra' component present during growth in EMM-G was very similar to that for the wild-type. Assigning the uptake rate during growth in EMM-A to system I and the rate enhancement during growth in EMM-G to system II is entirely consistent with the model presented above.

Strain ED 429 showed a reduced level of uptake of arginine during growth in EMM-A or EMM-G. In both these cases the decrease relative to the wild-type was between 60 and $70 \%$. Thus ED 429 shows no specific loss of either uptake system, but an overall reduction. Possible reasons for this are discussed below. 
Table 4. Uptake of arginine by strains $972, E D 428$ and ED 460

Cells of the strains indicated were grown in EMM-A or EMM-G. Samples $(500 \mu \mathrm{l})$ were added to $2 \mu \mathrm{Ci}$ $\left[{ }^{3} \mathrm{H}\right]$ arginine and unlabelled arginine to a final concentration of $10 \mu \mathrm{M}$. Samples $(150 \mu \mathrm{l})$ were taken at 10 min intervals, washed and counted. Uptake followed linear kinetics in each case. Samples for the determination of cell number were taken at the start of labelling.

\begin{tabular}{lcc} 
& \multicolumn{2}{c}{$\begin{array}{c}\text { Uptake rate } \\
\left(10^{-3} \times \text { c.p.min }{ }^{-1} \text { per } 10^{6} \text { cells }\right) \\
\text { during growth on: }\end{array}$} \\
\cline { 2 - 2 } Strain & $\overbrace{\text { EMM-A }}$ & EMM-G \\
972 & 4.59 & 8.28 \\
ED 428 & 0.34 & 5.43 \\
ED 460 & 0.73 & 7.31
\end{tabular}

Table 5. Effect of various amino acids on the uptake of arginine

Cultures of strain 972 growing in EMM-A and strain ED 460 growing in EMM-G were assayed for arginine uptake rate. Labelling conditions were: $1 \mathrm{ml}$ cells added to $1 \mu \mathrm{Ci}\left[{ }^{3} \mathrm{H}\right]$ arginine plus either $1 \mu \mathrm{M}$ or $23 \mu \mathrm{M}$-unlabelled arginine final concentration, plus $10 \mu \mathrm{l}$ of $10 \mathrm{mM}$-L-amino acid solution. Uptake rates were calculated from the linear portions of the uptake curves (generally between 10 and $30 \mathrm{~min}$ ) and are expressed as percentages of the rates in the absence of any added amino acid. 'The absolute uptake rates for the control cultures (no additional amino acids included) of strain 972 in EMM-A were $1.3 \times 10^{3}$ c.p.m. $\min ^{-1}$ per $10^{6}$ cells for $1 \mu \mathrm{M}$-arginine and $1.2 \times 10^{3}$ c.p.m. $\mathrm{min}^{-1}$ per $10^{6}$ cells for $23 \mu \mathrm{M}$. Corresponding values for $\mathrm{ED} 460$ were $1.4 \times 10^{3}$ and $1.4 \times 10^{3}$ respectively.

\begin{tabular}{|c|c|c|c|}
\hline \multirow[b]{2}{*}{ Culture* } & \multirow[b]{2}{*}{ Amino acid } & \multicolumn{2}{|c|}{$\begin{array}{c}\text { Relative uptake }(\%) \\
\text { in the presence of } \\
\text { arginine at: }\end{array}$} \\
\hline & & $1 \mu \mathrm{M}$ & $23 \mu \mathrm{M}$ \\
\hline 972 in EMM-A (system I) & $\begin{array}{l}\text { None } \\
\text { L-Ornithine. } \mathrm{HCl} \\
\text { L-Lysine. } \mathrm{HCl} \\
\text { L-Canavanine. } \mathrm{HCl} \\
\text { L-Tryptophan } \\
\text { L-Leucine }\end{array}$ & $\begin{array}{r}100 \\
55 \\
36 \\
41 \\
129 \\
112\end{array}$ & $\begin{array}{r}100 \\
58 \\
41 \\
42 \\
86 \\
63\end{array}$ \\
\hline ED 460 in EMM-G (system II) & $\begin{array}{l}\text { None } \\
\text { L-Ornithine. } \mathrm{HCl} \\
\text { L-Lysine. } \mathrm{HCl} \\
\text { L-Canavanine. } \mathrm{HCl} \\
\text { L-Tryptophan } \\
\text { L-Leucine }\end{array}$ & $\begin{array}{r}100 \\
69 \\
62 \\
81 \\
92 \\
82\end{array}$ & $\begin{array}{r}100 \\
91 \\
102 \\
114 \\
127 \\
141\end{array}$ \\
\hline
\end{tabular}

* Presumptive arginine uptake system functional shown in parentheses.

Several other experiments were done in order to test further the hypothesis that two distinct uptake systems were capable of mediating the uptake of arginine. First, strains ED 428, ED 460 and the wild-type 972 were compared. Table 4 shows the relative rates of arginine uptake during growth in EMM-A or EMM-G. Under both sets of conditions the backcrossed derivative of ED 428, ED 460, showed a slightly higher level of uptake than ED 428, though still lower than the wild-type values. Other experiments (not shown) indicated that minor changes in the incubation conditions had no significant effect on the relative uptake rates of the strains. In all subsequent experiments the backcrossed derivative ED 460 was used.

To investigate further the difference between arginine uptake rate during growth on glutamate and on ammonium, shift experiments were done. Cells of 972 and ED 460 were grown in EMM-G; portions were added to $\left[{ }^{3} \mathrm{H}\right]$ arginine or to $\left[{ }^{3} \mathrm{H}\right]$ arginine plus ammonium chloride to bring the final concentrations to that in EMM-A. Samples taken at intervals to estimate arginine uptake showed that the rate of arginine uptake fell rapidly after addition of ammonium. In wild-type cells the reduction was by about $75 \%$, while the can 1.1 strain showed a more drastic reduction by nearly $90 \%$. The observed reductions in rate are presumably due to rapid inhibition 
Table 6. Properties of diploid strains heterozygous for can1.1

Diploid strains heterozygous canl.1/canl ${ }^{+}$and the corresponding homozygotes were tested for growth on $\mathrm{EMM}-\mathrm{G}$ in the presence of $\mathrm{L}$-canavanine. $\mathrm{HCl}$ at $10 \mu \mathrm{g} \mathrm{ml}^{-1}$. Colony diameter after $5 \mathrm{~d}$ growth was measured as described in Table 1. For arginine uptake determination, cells were grown in EMM-A at $30{ }^{\circ} \mathrm{C}$. Samples $(2 \mathrm{ml})$ were added to $2 \mu \mathrm{Ci}\left[{ }^{3} \mathrm{H}\right]$ arginine with a final arginine concentration of $23 \mu \mathrm{M}$.

$\begin{array}{ccc}\text { Strain } & \begin{array}{c}\text { Colony diameter } \\ (\mu \mathrm{m})\end{array} & \text { Arginine uptake } \\ { }_{\text {can }}{ }^{+} / \text {can }^{+} & \text {Single cells } & 6.01 \\ { }^{*} \text { can } 1.1 / \text { can } 1^{+} & 100 & 2.34 \\ { }^{*} \text { can } 1.1 / \text { can } 1^{+} & 100 & 3.54 \\ \text { can } 1.1 / \text { canl } 1.1 & 200-300 & 1.75\end{array}$

* Results for two independent diploids heterozygous for canl.

$\dagger$ Expressed as $10^{-3} \times$ c.p.m. min $^{-1}$ per $10^{6}$ cells.

of uptake system II by the presence of ammonium: in the can 1.1 strain the reduction is by almost $90 \%$ since system I operates at only a very low level. In the wild-type system I is still active and the reduction is less dramatic.

As stated above, rapid inhibition of system II is the most likely explanation for the rapid fall in uptake following the addition of ammonium. An alternative explanation might be that some component of system II whose synthesis is ammonium repressed is very labile and has a short turnover time in vivo.

Further evidence that systems I and II are physiologically distinct comes from experiments where the effect of various amino acids on arginine uptake was determined. To allow the maximum possible sensitivity in discriminating between the systems, wild-type cells growing in EMM-A (system I only) were compared with ED 460 cells growing in EMM-G (essentially only system II functional). The results (Table 5) show that presumptive system I is inhibited by basic amino acids such as lysine, ornithine or canavanine, but not significantly affected by tryptophan. Leucine showed some inhibition in one case. In contrast, system II showed little inhibition by any of the amino acids tested, and showed slight stimulation in the presence of tryptophan or leucine, at the higher arginine concentration tested (Table 5).

\section{Dominance relationships for can 1.1}

It has been argued so far that the $\mathrm{canl}^{+}$gene codes for a component of an uptake system for arginine and canavanine. Both genetical and physiological data suggest that in the can1.1 strain ED 460, one uptake system is defective. According to this hypothesis a diploid strain heterozygous for canl.1 would be expected to show a rate of arginine uptake midway between those of the corresponding homozygotes, as in Sacch. cerevisae (Grenson et al., 1966), assuming the can 1 product to be limiting. Results for the two homozygotes and two independent heterozygotes are shown in Table 6. While the heterozygote values clearly fall between those for the homozygotes, the former are closer to the mutant homozygote than to the wild-type homozygote. Other experiments (not shown) indicated that minor changes in the incubation conditions had no significant effect on the relative uptake rates of the strains.

The diploid strains were examined for their sensitivity to canavanine by replicating actively growing cells onto the surface of EMM-G containing canavanine. The wild-type diploid showed no growth. The mutant homozygote grew, though not as strongly as the corresponding haploid mutant. The heterozygotes showed some growth, though this was weaker than that of the mutant homozygote. Within the patches of poorly growing heterozygous diploids faster growing colonies were observed: these presumably resulted from cells which had undergone mitotic crossing-over and become homozygous for the canl.l allele. To obtain a better estimate of the extent of inhibition by canavanine, the strains were streaked for single colonies onto EMM-G plus canavanine $\left(10 \mu \mathrm{g} \mathrm{ml}^{-1}\right)$. After $5 \mathrm{~d}$ growth the homozygous can 1.1 diploid showed better growth than the heterozygotes (Table 6), while the wild-type diploid showed no growth.

In conclusion, diploids heterozygous canl.1/canl ${ }^{+}$show uptake and resistance phenotypes intermediate between those of the two homozygotes. 


\section{DISCUSSION}

We describe in this paper an investigation into the mechanisms of canavanine resistance and arginine uptake in the fission yeast $S$. pombe. The strains we obtained from Bern as 'can1.I' (ED 428) and 'can 2.l' (ED 429) both appear to be double mutant strains, each carrying a major canavanine resistance mutation and an additional modifier or enhancer mutation. It is not certain whether ED 428 and ED 429 share the same modifier mutation. In the case of ED 428 the modifier, provisionally designated canx, increases the level of resistance to canavanine during growth with glutamate as nitrogen source, but not with ammonium (Table 1, compare ED 428 and ED 460).

Two lines of evidence lead to the conclusion that the canl $1^{+}$gene product is required for activity of one arginine uptake system, referred to as system I. First, the inability of arg canl.I double mutants to grow on EMM-A plus arginine (Table 3 ) is consistent with an uptake defect. Second, direct measurements in canl.l and canl ${ }^{+}$strains (Table 4) show that the presence of canl.1 reduces the rate of arginine uptake substantially. During growth on ammonium the reduction is about $90 \%$, while a smaller reduction was found for cells growing on glutamate. It is likely that the can $1^{+}$gene itself codes for a component of arginine uptake system I, though the possibility that the gene has a regulatory rather than structural role cannot be excluded at present.

There is a substantial difference in the rates of arginine uptake between cells growing on ammonium and cells growing on glutamate (Fig. 1, Table 4). This difference, particularly marked in canl.1 strains, points strongly to the existence of a second system which can mediate the uptake of arginine. This system, system II, appears to be strongly inhibited or repressed by ammonium (Table 4). System I is inhibited by basic amino acids while system II is not (Table 5), suggesting that system I may be a permease primarily concerned with basic amino acid uptake, similar to the CANI system in Sacch. cerevisiae (Grenson et al., 1966; Whelan et al., 1979). The physiological role of system II is uncertain, though its inhibition or repression by ammonium shows clear parallels with the general amino acid uptake system in Sacch. cerevisiae (Grenson et al., 1966, 1970). It may be significant that ED 460 (can1.1) shows the same level of canavanine resistance as ED 428 (canl.1 canx) during growth with ammonium as nitrogen source, but during growth on glutamate ED 428 is significantly more resistant (Table 1). It is possible that canx is defective in an element of uptake system II. The rather higher rate of arginine uptake by ED 460 compared with ED 428 (Table 4) is consistent with this proposal.

The nature of the defect in ED 429 is uncertain. This strain grows slightly more slowly than either wild-type or canl strains in the absence of canavanine (Table 1). Arginine uptake is reduced by about $60 \%$ compared with the wild-type, irrespective of the nitrogen source (Fig. 1). There is no genetic interaction between can2.1 and arg mutations, which is expected since arginine uptake is only incompletely blocked. It is possible that can 2.1 is defective in uptake of many substrates in the same way as $c y h 3$ and cyh 4 in $S$. pombe (Johnston \& Coddington, 1983), or the aap and apf mutants of Sacch. cerevisiae (Surdin et al., 1965; Grenson \& Hennaut, 1971).

In conclusion, there are strong similarities between the mechanisms of genetic resistance to canavanine in $S$. pombe and in Sacch. cerevisiae. A mutation in a single major gene, designated canl in both yeasts, drastically reduces the activity of an uptake system with specificity for arginine and other basic amino acids. A second system can mediate arginine uptake in both organisms, and this system is strongly inhibited during growth on ammonium. In Sacch. cerevisiae this system (coded by the GAP gene) also mediates the uptake of many amino acids of various types: in $S$. pombe the genetic basis of the system is uncertain, though it is possible that can2.1 is defective in it. Further work will be required to elucidate this.

In $S$. pombe as in Sacch. cerevisiae, arg canl double mutants are unable to utilize exogenous arginine under conditions of nitrogen repression. This should permit selection in $S$. pombe both for $\mathrm{canl}^{+}$and for canl genotypes. The latter can be selected by growth in the presence of canavanine, while the former should be favoured by selecting for growth of an arginine auxotroph in the presence of ammonium. 
We would like to express our thanks to Yvonne Bisset and Joyce Shannan for expert technical assistance, and to Murdoch Mitchison for critical reading of the manuscript. The figure was carefully drawn by Yvonne Bisset. This work was supported by the Science and Engineering Research Council.

\section{REFERENCES}

Beach, D. \& Nurse, P. (1981). High frequency transformation of the fission yeast Schizosaccharomyces pombe. Nature, London 290, 140-142.

COOPER, T. G. (1982). Transport in Saccharomyces cerevisiae. In The Molecular Biology of the Yeast Saccharomyces, vol. II, pp. 399-461. Edited by J. Strathern, E. Jones \& J. Broach. New York: Cold Spring Harbor Laboratory.

Creanor, J. \& Mitchison, J. M. (1982). Patterns of protein synthesis during the cell cycle of the fission yeast Schizosaccharomyces pombe. Journal of Cell Science 58, 263-285.

Grenson, M. \& Hennaut, C. (1971). Mutation affecting activity of several distinct amino acid transport systems in Saccharomyces cerevisiae. Journal of Bacteriology 105, 477-482.

GRENSON, M. \& HoU, C. (1972). Ammonia inhibition of the general amino acid permease and its suppression in the NADPH-specific glutamate dehydrogenaseless mutants of Saccharomyces cerevisiae. Biochemical and Biophysical Research Communications 48, 749-756.

Grenson, M., Mousset, M., Wiame, J.-M. \& Bechet, J. (1966). Multiplicity of the amino acid permeases in Saccharomyces cerevisiae. I. Evidence for a specific arginine-transporting system. Biochimica et biophysica acta 127, 325-338.

Grenson, M., Hou, C. \& Crabeel, M. (1970). Multiplicity of the amino acid permeases in Saccharomyces cerevisiae. IV. Evidence for a general amino acid permease. Journal of Bacteriology 103, 770-777.

Gutz, H., Heslot, H., Leupold, U. \& Loprieno, N.
(1974). Schizosaccharomyces pombe. In Handbook of Genetics, pp. 395-446. Edited by R. C. King. New York: Plenum Press.

Gygax, A. \& Thuriaux, P. (1984). A revised chromosome map of the fission yeast Schizosaccharomyces pombe. Current Genetics 8, 85-92.

Johnston, P. A. \& Coddington, A. (1983). Multiple drug resistance in the fission yeast Schizosaccharomyces pombe: correlation between drug and amino acid uptake and membrane ATPase activities. Current Genetics 7, 299-307.

Kohli, J., Hottinger, H., Munz, P., Strauss, A. \& Thuriaux, P. (1977). Genetic mapping in Schizosaccharomyces pombe by mitotic and meiotic analysis and induced haploidization. Genetics 87, 471489.

Mitchison, J. M. (1970). Physiological and cytological methods for Schizosaccharomyces pombe. Methods in Cell Physiology 4, 131-165.

STRAuss, A. (1979). Isolation and genetical classification of aromatic amino acid auxotrophic mutants in Schizosaccharomyces pombe. Journal of General Microbiology 113, 173-176.

Surdin, Y., Sly, W., Sire, J., Bordes, A. M. \& DeRobichon-Szulmajster, H. (1965). Propriétés et contrôle génétique du système d'accumulation des acides amines chez Saccharomyces cerevisiae. Biochimica et biophysica acta 107, 546-566.

Whelan, W. L., Gocke, E. \& Manney, T. R. (1979). The CAN 1 locus of Saccharomyces cerevisiae: fine structure analysis and forward mutation rates. Genetics 91, 35-51. 